\title{
Assessment of The Frequency and Correlation of Carotid Artery Calcifications and Pulp Stones with Idiopathic Osteosclerosis Using Digital Panoramic Radiographs
}

\author{
Sema Sonmez Kaplan (), Tuna Kaplan (1), Guzide Pelin Sezgin 1 \\ Department of Endodontics, Faculty of Dentistry, Biruni University, Istanbul, Turkey. \\ Correspondence Author: Sema Sonmez Kaplan \\ E-mail: semak@biruni.edu.tr
}

Received: $15.12 .2020 \quad$ Accepted: 25.03 .2021

\begin{abstract}
Objective: The aim of this study was to assess the correlation of carotid artery calcifications (CACs) and pulp stones with idiopathic osteosclerosis (IO) using digital panoramic radiographs (DPRs) to determine whether pulp stones or IO might be possible indicators of the presence of CACs.

Methods: In total, DPRs of 1207 patients (645 females and 562 males) taken within 2018 were retrospectively evaluated to determine the prevalence of CACs, pulp stones and IO according to age and sex. Statistical analysis was performed using chi-square test and Fisher's exact chisquare test.

Results: In total, 287 (23.8\%) patients had at least one pulp stone, and 64 (5.3\%) patients had CACs. The negative/negative (-/-) status of CACs/ pulp stones was significantly higher in the $18-29$ years age group than in the $30-39,40-49,50-59$ and $\geq 60$ years age groups ( $p<0.05)$. It was also significantly higher in males than females $(p<0.05)$. Sixteen $(1.3 \%)$ patients had IO, which was related to right mandibular molars in all cases. Patients with CACs had a significantly higher prevalence of $\mathrm{IO}(6.3 \%)$ than those without CACs $(1 \%)(p<0.05)$. There was no statistically significant association between pulp stones and the presence of IO and CACs $(p>0.05)$.

Conclusion: Within the limitations of this study, pulp stones were not found to be diagnostic indicators of CACs. However, the presence of IO might be a risk factor for CACs.

Keywords: Carotid artery calcification, dental pulp stone, digital panoramic radiograph, idiopathic osteosclerosis.
\end{abstract}

\section{INTRODUCTION}

Calcium mineral deposition in ectopic areas and dystrophic lesions can result in pathological conditions, such as arterial atherosclerosis. This mineral deposition occurs in the presence of lesions or plaques, which can be evaluated radiographically. The structure underlying the atherosclerotic calcification shows a connection between bone biology and chronic plaque inflammation, passive mineral deposition and bone formation or remodelling (1).

Although calcification of atherosclerotic lesions is common (1), some previous reports found that the existence and severity of calcification, in addition to long-term vascular events, were correlated with a patient's entire burden of atherosclerotic lesions $(2,3)$. Digital panoramic radiography is one of the most widely used diagnostic imaging methods in dentistry. Due to their wide coverage of the head and neck region, digital panoramic radiographs (DPRs) can capture a large amount of data in a single image. The low radiation dose of DPRs also makes them highly suitable for use in dental clinics (4).
Any calcifications within the lumen of the carotid artery appear as radiopacities on high-quality DPRs. Detection of carotid artery calcifications (CACs) in DPRs is vital due to the significant morbidity and mortality attributable to atherosclerotic lesions (5).

Several studies have examined the effectiveness of radiographic evaluations of CACs using DPRs in various populations $(6,7)$. One study reported that the advantages of digital panoramic radiography for assessing CAC lesions included its non-invasiveness and cost effectiveness (8). Another study concluded that dentists who detect CACs in patient's DPRs should consider a prophylactic specialist examination (9). Following a radiographic diagnosis of CACs, various other diagnostic imaging modalities, such as ultrasonography, magnetic resonance imaging, computed tomography and angiography, with higher resolution capacities are often needed to validate the diagnosis. Following the detection of CACs, a previous study indicated that prompt interventions were needed to combat associated adverse vascular events (10). 
Pulp stones are dystrophic calcifications. Such calcifications may be found in both the coronal and radicular parts of the dental pulp cavity (11). Previous studies demonstrated the ability of both dental radiographs and cone beam computed tomography to detect pulp stones (12-14). Although the etiology of pulp stones is considered idiopathic, some risk factors have been proposed: pulp degeneration, aging, genetics, chronic irritants, trauma, atherosclerosis, cardiovascular diseases, kidney diseases, gallstones and salivary gland stones (15). A previous study suggested that a high level of blood calcium or metabolic dysfunction could be considered etiologies for pulp stones, in the same way that calcifications play a role in the etiology of atherosclerosis (16). Based on a strong correlation between pulp stones and CACs, Yeluri et al. (17) suggested that further evaluations of CACs were required in patients with multiple pulp stones.

Idiopathic osteosclerosis (IO) refers to localized areas of compact bone that evolves within cancellous bone with an unidentified origin. $I O$ is asymptomatic, observed with different shapes and sizes found in both the mandible and maxilla, with a higher frequency in mandibular premolar and molar areas (18). The radiopacity in 10 can resemble condensing osteitis, cementoblastomas, hypercementosis, complex odontomas or focal cemento-osseous dysplasia, which are other pathologies of the jaws (19). The diagnosis is established based on an examination of the lesion morphology, along with clinical symptoms and radiographs.

It is unclear whether CACs are associated with an elevated risk of cardiovascular diseases (CVDs). Balbay et al. (20) concluded that the current burden of CVDs in Turkey was significant and that it was projected to increase due to the aging of a large part of the population. They also noted that CVDs were important causes of morbidity, premature mortality and disability in Turkey. Many patients may visit a dentist more frequently than they visit a physician. Dentists can play a role in the early diagnosis of CVDs by detecting the presence of pulp stones and IO on radiographic examinations.

To our knowledge, no study has examined the correlation between CACs, pulp stones and 10 in the adult Turkish population. The objective of this study was to assess the potential correlation between the presence of CACs, pulp stones and $\mathrm{IO}$ in males and females of different age groups to determine whether pulp stones or 10 might be a significant indicator of the presence of CACs.

\section{MATERIALS AND METHODS}

\subsection{Sample Selection}

This cross-sectional study used DPRs from patients who visited Biruni University Faculty of Dentistry for dental treatment between January 2018 and December 2018. In total, 2108 DPRs were examined. Of these, the DPRs of 1207 patients ( 645 females and 562 males aged 18 years or older) fulfilled the following inclusion criteria: for diagnostic purposes, each DPR had to clearly show maxillary and mandibular teeth, in addition to the complete area posterior to the angle of the mandible at the level of the C3-C4 cervical vertebrae, with optimum contrast and density and with no distortion or superimposition of anatomic structures. Low-quality radiographs with incorrect exposure times or angulations were excluded. Regarding the pulp stone evaluation, teeth with fractures, caries and root canal fillings were excluded. The exclusion criteria for the 10 evaluations were as follows: condensing osteitis associated with chronic inflammation (teeth with caries, deep restorations or root canal obturations, etc.), mixed radiopaque and radiolucent lesions, benign fibro-osseous lesions, and tori or exostosis. The DPR of each patient that fulfilled the study requirements was evaluated and demographic data in terms of age and sex were documented.

This study complied with the tenets of the World Medical Association Declaration of Helsinki and was approved by the ethics committee of Biruni University (2020/37-16).

\subsection{Radiographic Evaluation}

A dental radiology assistant took all the panoramic radiographs using a Sirona Galileos panoramic X-ray unit (Sirona, Bensheim, Germany). Each radiograph was analyzed using Picture Archiving and Communication Systems (PACS) software, Version 1.1.1.6 for Windows 10 (Microsoft Corporation, Redmont, WA, USA), on a 28-in Samsung LU28H750UQMXUF monitor (Samsung Electronics, Seoul, South Korea) with $3,840 \times 2,160$ pixel resolution. Each radiograph was assessed as follows: All original digital images were magnified using the magnification function of the PACS software. The examiner manipulated the images to improve their contrast and brightness to give subjectively the clearest images in the analyzed areas.

One endodontist and one dental radiologist, each of whom had at least 5 years of experience, assessed each image. Before the evaluation of the radiographs, each examiner assessed a series of 30 radiographs that were not associated with the present study for calibration between the examiners. Any disagreement between the examiners in the evaluation was resolved through discussion until agreement was reached. Cohen's kappa was used for inter-observer consensus, which corresponded to very good agreement (value of 0.86 ).

The diagnosis of CACs was based on heterogeneous unilateral or bilateral nodular, punctate and vertico-linear radiopacities detected posterio-inferior to the angle of the mandible at the level of the C3-C4 cervical vertebrae (Figure 1). The presence of idiopathic pulp stones was characterized as clear radiopaque masses positioned in the coronal pulp chamber, as seen in Figure 1 and Figure 2. Based on these radiological criteria, IO was characterized as well-defined, ovoid, round or irregularly shaped and radiopaque lesions associated with normal bone, with no radiolucent surroundings (Figure 1). 


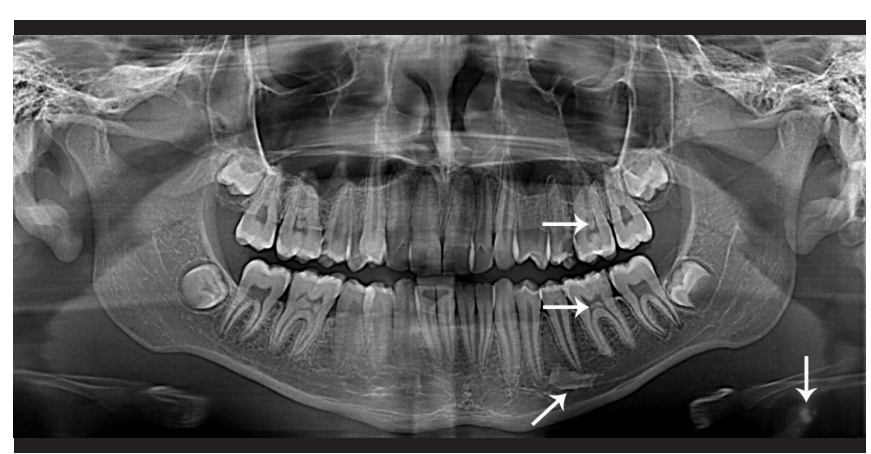

Figure 1. Panoramic radiograph showing a detectable carotid artery calcification on the left and idiopathic pulp stones within the coronal pulp chambers of the maxillary and mandibular left first molars, with idiopathic osteosclerosis of the left mandibular premolar area (white arrows).

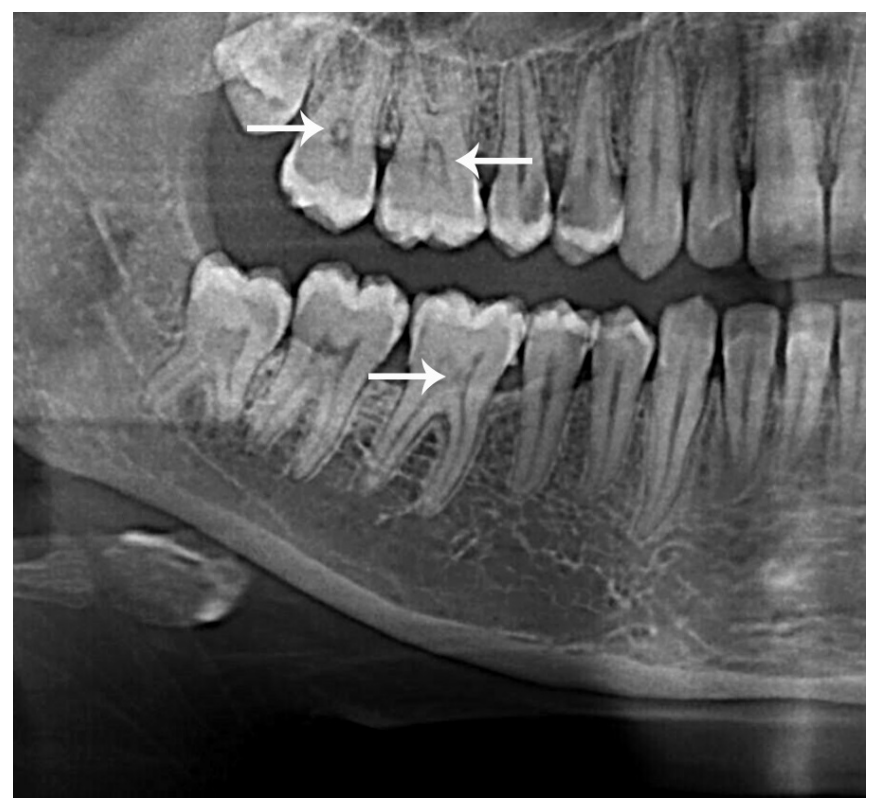

Figure 2. A close-up view of a panoramic radiograph showing idiopathic pulp stones within the coronal pulp chambers of the maxillary right first and second molars and mandibular right first molar (white arrows).

\subsection{Statistical Analysis}

Statistical analyses were performed using IBM SPSS Statistics 22 (IBM SPSS, Turkey). A chi-square test and Fisher's exact chi-square test were used to compare differences between the age and gender groups in respect of the presence of CACs, pulp stones and IO. The statistical significance level was set at $p<0.05$.

\section{RESULTS}

In total, DPRs of 1207 patients were examined. The mean \pm standard deviation (SD) age of the patients was $38.15 \pm$ 12.96 years. In the study group, 645 (53.4\%) patients were females, and 562 (46.6\%) were males. In total, 64 (5.3\%) patients had CACs: $53.1 \%$ on the right, $21.9 \%$ on the left and
$25 \%$ bilaterally. 287 (23.8\%) patients had at least one pulp stone and $16(1.3 \%)$ patients had 10 , which was related to right mandibular molars in all cases. The presence of CACs according to age was statistically significant $(p=0.002)$. The percentage of patients with CACs in the $\geq 60$ years age group was significantly higher (12.2\%) than that in the 18-29 (3\%), $40-49(3.5 \%)$ and $50-59(4.9 \%)$ years age groups $(p=0.001$, $p=0.003$ and $p=0.047$, respectively). The frequency of CACs was significantly higher in the $30-39$ years $(7.1 \%)$ age group than in the $18-29(3 \%)$ and $40-49$ years (3.5\%) age groups ( $p=0.021$ and $p=0.045$ ). Furthermore, the frequency of CACs was significantly higher in females $(7.4 \%)$ than in males (2.8\%) $(p=0.001)$ (Table 1$)$.

Table 1. Descriptive analysis of age and sex and the relation to carotid artery calcifications

\begin{tabular}{|l|c|c|c|c|}
\hline & & \multicolumn{2}{|c}{ CACs } & \multirow{2}{*}{$p$} \\
\hline & & Absent & Present & \\
\hline & & $\mathrm{n}(\%)$ & $\mathrm{n}(\%)$ & \\
\hline & $18-29$ & $325(97)$ & $10(3)$ & $0.002^{1}$ \\
\hline Age & $30-39$ & $339(92.9)$ & $26(7.1)$ & \\
\hline & $40-49$ & $276(96.5)$ & $10(3.5)$ & \\
\hline & $50-59$ & $117(95.1)$ & $6(4.9)$ & \\
\hline Sex & $60+$ & $86(87.8)$ & $12(12.2)$ & \\
\hline & Female & $597(92.6)$ & $48(7.4)$ & $0.001^{2}$ \\
\hline & Male & $546(97.2)$ & $16(2.8)$ & \\
\hline
\end{tabular}

${ }^{1}$ Chi-Square test; ${ }^{2}$ continuity (yates) test; $p<0.05$ is statistically significant; CACs: carotid artery calcifications

The frequency of pulp stones according to age was statistically significant $(p<0.001)$. The frequency of pulp stones in the 1829 years age group was significantly lower $(15.8 \%)$ than that in the 30-39 (31.2\%), 40-49 (25.2\%) and 50-59 (25.2\%) years age groups ( $p<0.001, p=0.004$ and $p=0.021$, respectively). In addition, the frequency of pulp stones was significantly lower in the $\geq 60$ years age group (17.3\%) than in the $30-39$ years $(31.2 \%)$ age group $(p=0.010)$. The frequency of pulp stones was significantly higher in females $(28.5 \%)$ than in males $(18.3 \%)(p<0.001)$ (Table 2).

Negative/negative (-/-) status for CACs/pulp stones in the 18-29 years age group was significantly higher than that in the $30-39,40-49,50-59$ and $\geq 60$ years age groups ( $p<0.001$, $p=0.027, p=0.028$ and $p=0.001$, respectively). The negative/ positive $(-/+)$ status for CACs/pulp stones in the 30-39 years age group was significantly higher than that in the 40-49 and $\geq 60$ years age groups ( $p=0.002$ and $p=0.010)$. The positive/ negative (+/-) status for CACs/pulp stones in the 40-49 years age group was significantly lower than that in the $\geq 60$ years age group $(p=0.001)$. The $-/+$ status for CACs/pulp stones was significantly higher in females than in males, and the - / - status for CACs/pulp stones in males was significantly higher than that in females $(p<0.001)$ (Table 3$)$. 
The presence of pulp stones was not significantly associated with the presence of CACs ( $p>0.05)$ (Table 4).

The presence of 10 was significantly more common among patients with CACs than without CASs ( $6.3 \%$ vs. $1 \%)(p=0.008)$. On the other hand, there was no significant association between the presence of pulp stones and the presence of IO ( $p>0.05)$ (Table 5).

Table 2. Descriptive analysis of age and sex, and the relation to pulp stones

\begin{tabular}{|c|c|c|c|c|}
\hline & & \multicolumn{2}{|c|}{ Pulp Stones } & \multirow{3}{*}{$\boldsymbol{p}^{1}$} \\
\hline & & Absent & Present & \\
\hline & & n (\%) & n (\%) & \\
\hline & $18-29$ & $282(84.2)$ & $53(15.8)$ & $<0.001$ \\
\hline & 30-39 & $251(68.8)$ & $114(31.2)$ & \\
\hline \multirow[t]{3}{*}{ Age } & $40-49$ & $214(74.8)$ & $72(25.2)$ & \\
\hline & $50-59$ & $92(74.8)$ & $31(25.2)$ & \\
\hline & $60+$ & 81 (82.7) & $17(17.3)$ & \\
\hline \multirow[t]{2}{*}{ Sex } & Female & $461(71.5)$ & $184(28.5)$ & $<0.001$ \\
\hline & Male & 459 (81.7) & 103 (18.3) & \\
\hline
\end{tabular}

${ }^{1}$ Chi-Square test; $p<0.05$ is statistically significant

Table 3. Descriptive analysis of age and sex, and the association between pulp stones and carotid artery calcifications

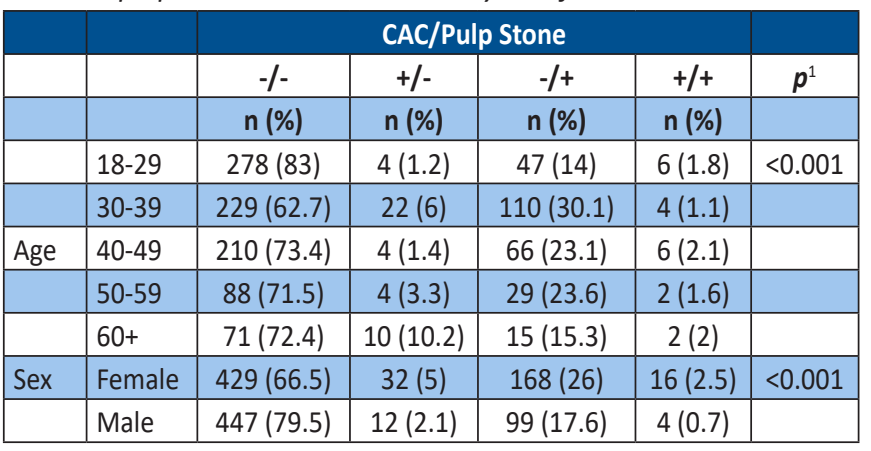

${ }^{1}$ Chi-Square test; $p<0.05$ is statistically significant; CACs: carotid artery calcifications

Table 4. Associations between the presence or absence of carotid artery calcifications and pulp stones

\begin{tabular}{|l|l|c|c|c|}
\hline & & \multicolumn{3}{|c|}{ CACs } \\
\hline & & Absent (-) & Present (+) & Total \\
\hline Pulp Stones & Absent (-) & $\mathbf{n}(\%)$ & $\mathrm{n}(\%)$ & $\mathbf{n}(\%)$ \\
\hline & Present (+) & $267(22.6)$ & $44(3.6)$ & $920(76.2)$ \\
\hline & Total & $1143(94.7)$ & $64(1.7)$ & $287(23.8)$ \\
\hline & $\boldsymbol{p}^{1}$ & & 0.149 & $1207(100)$ \\
\hline
\end{tabular}

${ }^{1}$ Chi-Square test; $p<0.05$ is statistically significant; CACs: carotid artery calcifications
Table 5. Associations between the presence or absence of idiopathic osteosclerosis (IO) and pulp stones and carotid artery calcifications (CACS)

\begin{tabular}{|l|l|c|c|c|}
\hline & & \multicolumn{2}{|c|}{ lO } & \multirow{2}{*}{$\boldsymbol{p}^{1}$} \\
\cline { 1 - 4 } & & Absent & Present & \\
\hline & & $\mathrm{n}(\%)$ & $\mathrm{n}(\%)$ & \\
\hline & Absent & $1131(99)$ & $12(1)$ & 0.008 \\
\hline & Present & $60(93.8)$ & $4(6.3)$ & \\
\hline Pulp Stones & Absent & $906(98.5)$ & $14(1.5)$ & 0.385 \\
\hline & Present & $285(99.3)$ & $2(0.7)$ & \\
\hline
\end{tabular}

${ }^{1}$ Fisher's Exact test; $p<0.05$ is statistically significant; 10: idiopathic osteosclerosis; CACs: carotid artery calcifications

\section{DISCUSSION}

The present study examined the frequency of CACs observed on DPRs in a Turkish population. Many reports have cited the use of DPRs as a valid approach to detect the presence of CACs $(2,6)$. Previous research noted a very high and positive correlation between ultrasonography, Doppler sonography and panoramic radiography in the identification of CACS $(2,6,21)$. Other DPR studies confirmed that CACs could serve as useful indicators of major cardiovascular and arterial diseases $(21,22)$. In the present study, we examined the association of CACs with pulp stones and IO using DPRs. The prevalence of CACs in the DPRs was $5.3 \%$ in the present study. Alzoman et al. (23) found a similar prevalence of CACs $(5 \%)$ in the Saudi population. The frequency of CACs in other studies varied from $2 \%$ to $6 \%$, with the frequency altering, depending on the study population's sex, age, ethnic background and life style (24-26). In the present study, the frequency of CACs was higher in females than in males. This finding was similar to that of Santos et al. (24) and Nasseh and Aoun (27). In our study, the highest frequency of CACs was in the oldest age group. This result was consistent with that of previous studies, which found that generally, CACs appeared to be more common in older age groups (both females and males) and that atherosclerosis was common in older people $(24,28)$. In the present study, we detected pulp stones in $23.8 \%$ of the patients. This result was higher, lower or close to that of the literature (29-31). The frequency of pulp stones was between 9.9 and $85 \%$ some previous studies $(30,32)$. This variation in the results might be due to a number of factors, such as the design of the studies and selection of the cases. In the present study, the frequency of pulp stones in females was significantly higher as compared with that in males. Consistent with our study results, Turkal et al. (33) and Sener et al. (34) found a significantly higher prevalence of pulp stone in females. An increase in the frequency of bruxism in females, as well as sex-related differences in oral care, with females more likely to have restorations and therefore long-term pulpal irritation, can explain these 
findings. In contrast to our results, Tassoker et al. (12) found no statistically significant difference in the frequency of pulp stones according to sex. Furthermore, previous studies reported no correlation between age and pulp stones $(34,35)$, whereas we detected the lowest frequency of pulp stones in the youngest age group (i.e. 18-29 years). Factors related to pulp stone formation, such as restorations, caries and parafunctional habits, all of which increase with age according to previous research (34), can explain the low pulp stone prevalence in the youngest age group in this study.

One of the primary aims of the present study was to evaluate the co-occurrence of pulp stones and CACs and to determine whether these anomalies could aid the early diagnosis of other medical conditions, such as CVDs. The results of our study revealed no positive correlation between pulp stones and CACs, in accordance with the finding of similar previous studies $(8,36,37)$. In contrast, a previous study reported a positive correlation between pulp stones, CACs and renal calcifications (17). The differences among these study results may be explained by mechanisms of pathogenesis. The fact that we did not record the presence of CVDs or other medical conditions may also explain the differences.

In the present study, the - / - status for pulp stones/CACs was higher in 18-29 y age group than in the other age groups. Horsley et al. (8) reported that the positive/positive $(+/+)$ status for pulp stones/CACs was higher in those aged 60 $y$ and older than in younger age groups. This finding was in accordance with our result.

Various studies have employed different imaging methods to investigate the 10 distribution in different populations $(38,39)$. These studies reported an 10 prevalence of between $1.8 \%$ and $7.6 \%$, which was higher than that found in our study $(1.3 \%)$. Variation in the population number, study designs and evaluation criteria might account for the reported differences in 10 prevalence. The etiology and pathways of $\mathrm{IO}$ are thought to be different than those of pulp stones and CACs (40). Nevertheless, according to our findings, there was a positive correlation between the presence of 10 and CACs. This result might contribute to further studies, as, to our knowledge, no published studies have investigated the correlation between these parameters.

A major limitation of this study was the use of DPRs for CAC examinations rather than carotid artery ultrasound, which superior to DPRs in terms of its sensitivity and specificity. An additional limitation is the debate about whether the CACs are associated with an elevated risk of CVDs.

\section{CONCLUSION}

The results of our study showed that CACs were more common in females, with an increased prevalence among males and females those aged $>60$ years. Pulp stones were more common in females. In terms of age, they were more common among individuals aged 30-39 years. According to our findings, pulp stones do not serve as a diagnostic component for CACs. However, the presence of 10 might be considered a risk factor for CACs. Further studies are needed to evaluate potential correlations among these parameters using different imaging techniques. As DPRs are a fundamental diagnostic component of routine dental examinations, where CACs are detected on DPRs, dentists may refer patients to a specialist for further investigations of possible atherosclerotic diseases.

\section{REFERENCES}

[1] Monteiro IA, Ibrahim C, Albuquerque R, Donaldson N, Salazar $\mathrm{F}$, Monteiro L. Assessment of carotid calcifications on digital panoramic radiographs: Retrospective analysis and review of the literature. J Stomatol Oral Maxillofac Surg 2018;119(2):102106.

[2] Garo M, Johansson E, Ahlqvist J, Levring Jaghagen E, Arnerlöv C, Wester P. Detection of calcifications in panoramic radiographs in patients with carotid stenoses $\geq 50 \%$. Oral Surg Oral Med Oral Pathol Oral Radiol 2014;117(3):385-391.

[3] Gustafsson N, Ahlqvist JB, Näslund U, Wester P, Buhlin K, Gustafsson A, Levring Jaghagen E. Calcified carotid artery atheromas in panoramic radiographs are associated with a first myocardial infarction: A case-control study. Oral Surg Oral Med Oral Pathol Oral Radiol 2018;125(2):199-204.

[4] Shah N, Bansal N, Logani A. Recent advances in imaging technologies in dentistry. World J Radiol 2014;6(10):794-807.

[5] Friedlander AH, Lande A. Panoramic radiographic identification of carotid arterial plaques. Oral Surg Oral Med Oral Pathol 1981;52(1):102-104.

[6] Christou P, Leemann B, Schimmel M, Kiliaridis S, Muller F. Carotid artery calcification in ischemic stroke patients detected in standard dental panoramic radiographs-A preliminary study. Adv Med Sci 2010;55(1):26-31.

[7] Alman AC, Johnson LR, Calverley DC, Grunwald GK, Lezotte LC, Hokanson JE. Validation of a method for quantifying carotid artery calcification from panoramic radiographs. Oral Surg Oral Med Oral Pathol Oral Radiol 2013;116(4):518-524.

[8] Horsley SH, Beckstrom B, Clark SJ, Scheetz JP, Khan Z, Farman AG. Prevalence of carotid and pulp calcifications: a correlation using digital panoramic radiographs. Int J Comput Assist Radiol Surg 2009;4(2):169-173.

[9] Brito AC, Nascimento HA, Argento R, Beline T, Ambrosano GMB, Freitas DQ. Prevalence of suggestive images of carotid artery calcifications on panoramic radiographs and its relationship with predisposing factors. Cien Saude Colet 2016;21(7):22012208.

[10] Ertas ET, Sisman Y. Detection of incidental carotid artery calcifications during dental examinations: Panoramic radiography as an important aid in dentistry. Oral Surg Oral Med Oral Pathol Oral Radiol Endod 2011;112(4):11-17.

[11] Farman AG. Panoramic radiology and the detection of carotid atherosclerosis. Panor Imaging News 2001;1:1-6.

[12] Tassoker M, Magat G, Sener S. A comparative study of cone-beam computed tomography and digital panoramic radiography for detecting pulp stones. Imaging Sci Dent 2018;48(3):201-212.

[13] Hsieh CY, Wu YC, Su CC, Chung MP, Huang RY, Ting TY, Lai CK, Chang KS, Tsai YWC, Shieh YS. The prevalence and distribution of radiopaque, calcified pulp stones: a cone-beam computed 
tomography study in a northern Taiwanese population. J Dent Sci 2018;13(2):138-144.

[14] Patil SR, Ghani HA, Almuhaiza M, AAl-Zoubi I, Anil KN, Misra M, Raghuram PH. Prevalence of pulp Stones in a Saudi Arabian subpopulation: a cone-beam computed tomography study. Saudi Endod J 2018;8(2):93-98.

[15] Nayak M, Kumar J, Prasad LK. A radiographic correlation between systemic disorders and pulp stones. Indian J Dent Res 2010;21(3):369-373.

[16] Khojastepour L, Bronoosh P, Khosropanah S, Rahimi E. Can dental pulp calcification predict the risk of ischemic cardiovascular disease? J Dent (Tehran) 2013;10(5):456-460.

[17] Yeluri G, Kumar CA, Raghav N. Correlation of dental pulp stones, carotid artery and renal calcifications using digital panoramic radiography and ultrasonography. Contemp Clin Dent 2015;6(1):147-151.

[18] Kawai T, Hirakuma H, Murakami S, Fuchihata H. Radiographic investigation of idiopathic osteosclerosis of the jaws in Japanese dental outpatients. Oral Surg Oral Med Oral Pathol Oral Radiol 1992;74(2):237-242.

[19] MacDonald-Jankowski DS. Idiopathic osteosclerosis in the jaws of Britons and of the Hong Kong Chinese: radiology and systematic review. Dentomaxillofac Radiol 1999;28(6):357363.

[20] Balbay $Y$, Gagnon-Arpin I, Malhan S, Öksüz ME, Sutherland G, Dobrescu A, Villa G, Ertuğrul G, Habib M. Modeling the burden of cardiovascular disease in Turkey. Anatol J Cardiol 2018;20(4):235-240.

[21] Abreu TQ, Ferreira EB, de Brito Filho SB, Desales KPP, Lopes $F F$, de Oliveria AEF. Prevalence of carotid artery calcifications detected on panoramic radiographs and confirmed by Doppler ultrasonography: Their relationship with systemic conditions. Indian J Dent Res 2015;26(4):345-350.

[22] Pornprasertsuk-Damrongsri S, Virayavanich W, Thanakun S, Siriwongpairat $P$, Amaekchok $P$, Khovidhunkit W. The prevalence of carotid artery calcifications detected on panoramic radiographs in patients with metabolic syndrome. Oral Surg Oral Med Oral Pathol Oral Radiol Endod 2009;108(4):57-62.

[23] Alzoman HA, Al-Sadhan RI, Al-Lahem ZH, Al-Salkaker AN, AlFawaz YF. Prevalence of carotid calcification detected on panoramic radiographs in a Saudi population from a training institute in Central Saudi Arabia. Saudi Med J 2012;33(2):177181.

[24] Santos JM, Soares GC, Alves AP, Kurita LM, Silva PGB, Costa FWG. Prevalence of carotid artery calcifications among 2,500 digital panoramic radiographs of an adult Brazilian population. Med Oral Patol Oral Cir Bucal 2018;23(3):256-261.

[25] Gonçalves JR, Yamada JL, Berrocal C, Westphalen FH, Franco $A$, Fernandes. Prevalence of pathologic findings in panoramic radiographs: Calcified carotid artery atheroma. Acta Stomatol Croat 2016;50(3):230-234.
[26] Lee JS, Kim OS, Chung HJ, Kim YJ, Kweon SS, Lee YH, Shin MH, Yoon SJ. The prevalence and correlation of carotid artery calcification on panoramic radiographs and peripheral arterial disease in a population from the Republic of Korea: The Dong-gu study. Dentomaxillofac Radiol 2013;42(3):29725099.

[27] Nasseh I, Aoun G. Carotid artery calcification: a digital panoramic-based study. Diseases 2018;6(1):15.

[28] Bayer S, Helfgen EH, Bös C, Kraus D, Enkling N, Mues S. Prevalence of findings compatible with carotid artery calcifications on dental panoramic radiographs. Clin Oral Investig 2011;15(4):563-569.

[29] Kannan S, Kannepady SK, Muthu K, Jeevan MB, Thapasum A. Radiographic assessment of the prevalence of pulp stones in Malaysians. J Endod 2015;41(3):333-337.

[30] Chandler NP, Pitt Ford TR, Monteith BD. Coronal pulp size in molars: a study of bitewing radiographs. Int Endod J 2003;36(11):757-763.

[31] Gulsahi A, Cebeci Al, Ozden S. A radiographic assessment of the prevalence of pulp stones in a group of Turkish dental patients. Int Endod J 2009;42(8):735-739.

[32] Tomczyk J, Komarnitki J, Zalewska M, Wisniewska E, Szopinski K, Olczyk-Kowalczyk D. The prevalence of pulp stones in historical populations from the middle Euphrates valley (Syria). Am J Phys Anthropol 2014;153(1):103-115.

[33] Turkal M, Tan E, Uzgur R, Hamidi MM, Colak H, Uzgur Z. Incidence and distribution of pulp stones found in radiographic dental examination of adult Turkish dental patients. Ann Med Health Sci Res 2013;3(4):572-576.

[34] Sener S, Cobankara FK, Akgünlü F. Calcifications of the pulp chamber: prevalence and implicated factors. Clin Oral Investig 2009;13(2):209-215.

[35] al-Hadi Hamasha A, Darwazeh A. Prevalence of pulp stones in Jordanian adults. Oral Surg Oral Med Oral Pathol Oral Radiol Endod 1998;86(6):730-732.

[36] Kansu Ö, Özbek M, Avcu N, Aslan U, Kansu H, Gençtoy G. Can dental pulp calcification serve as a diagnostic marker for carotid artery calcification in patients with renal diseases? Dentomaxillofac Radiol 2009;38(8):542-545.

[37] Patil S, Sinha N. Pulp stone, haemodialysis, end-stage renal disease, carotid atherosclerosis. J Clin Diag Res 2013;7(6):12281231.

[38] Li N, You M, Wang H, Ren J, Zhao S, Jiang M, Xu L, Liu Y. Bone islands of the craniomaxillofacial region. J Cranio Max Dis 2015;2(1):5-9.

[39] Verzak Z, Celap B, Modrić VE, Soric P, Karlovic Z. The prevalence of idiopathic osteosclerosis and condensing osteitis in Zagreb population. Acta Clin Croat 2012;51(4):573-577.

[40] Moshfeghi M, Azimi F, Anvari M. Radiologic assessment and frequency of idiopathic osteosclerosis of jawbones: an interpopulation comparison. Acta Radiol 2014;55(10):12391244. 